\title{
List of Conferences
}

(C) Marta Olivetti Belardinelli and Springer-Verlag 2007

2007

1st World Congress on Controversies in Neurology (CONy)

September 6-9, 2007; Berlin (Germany)

Info: http://www.comtecmed.com/cony

International Conference on Artificial Neural

Networks 2007

September 9, 2007; Porto (Portugal)

Info: http://www.icann2007.org

International Association for Research in Economic Psychology (IAREP) Annual Conference September 9-12, 2007; Ljubljana (Slovenia) Info: http://www.psy.ff.uni-lj.si/IAREP/

17th International Conference on Artificial Neural Networks (ICANN 2007)

September 9-13, 2007; Porto (Portugal)

Info: http://www.icann2007.org/

8th Workshop on Computational Logic in Multi-Agent Systems

September 10-11, 2007; Porto (Portugal)

Info: http://www.research.nii.ac.jp/climaVIII/

International Conference on Affective Computing and Intelligent Interaction

September 12-14, 2007; Lisbon (Portugal)

Info: http://www.gaips.inesc-id.pt/acii2007/index.html

Agency and Responsibility: Perspectives

from Metaphysics, Ethics, and the Emerging

Sciences of Brain and Behavior

September 13-15, 2007; Bloomington, Indiana (USA)

Info: http://www.indiana.edu/ agenresp
First European Conference on Social, Emotional and Behavioural Competence and Difficulties in Children and Young Persons

September 13-15, 2007; Malta (Malta)

Info: http://www.educ.um.edu.mt/sebcd

7th WSEAS International Conference on Signal, Speech and Image Processing

September 15-17, 2007; Beijing (China)

Info: http://www.wseas.org/conferences/2007/china/ssip

7th International Conference on Intelligent

Virtual Agents

September 17-19, 2007; Paris (France)

Info: http://www.iva07.ntua.gr

From molecules to cognition: A tribute to Jean-Pierre Changeux

September 17-19, 2007; Paris (France)

Info: http://www.pasteur.fr/infosci/conf/sb/ neuroscience_pasteur/

International Conference on Applied Psychology September 20-23, 2007; Iasi (Romania) Info: http://www.psih.uaic/ npaiasi07

Nature Inspired Machine Learning NIML'2007 September 25-30, 2007; Corfu (Greece) Info: http://www.ruta.co.uk/niml.pdf

18th International Conference on Algorithmic Learning Theory

October 1-4, 2007; Sendai, Japan

Info: http://www-alg.ist.hokudai.ac.jp/thomas/ALT07/ alt07.jhtml 
Questions of dispute. On the relationship of empirical research and anthropological theory in the beginning 21st century October 1-4, 2007; Halle (Germany) Info: http://www.dgv-tagung2007.de/

\section{Human Factors \& Ergonomics Society (HFES)} Annual Meeting

October 1-5, 2007; Baltimore, Maryland (USA)

Info: http://www.hfes.org/web/HFESMeetings/ meetings.html

32nd Annual Meeting of the Semiotic Society of America

October 4-7, 2007; New Orleans, Louisiana (USA)

Info: http://www.uwf.edu/tprewitt/SSA.htm

Workshop on Concepts, Essentialism, and Externalism

October 5-6, 2007; Turku (Finland)

Info: http://www.users.utu.fi/jusjyl/workshop.htm

2nd International Symposium on Brain, Vision and Artificial Intelligence (BVAI 2007)

October 10-12, 2007; Naples (Italy)

Info: http://www.bvai.cib.na.cnr.it/BVAI2007

2007 NeuroPsychoEconomics

October 14-16, 2007; Vienna (Austria)

Info: http://www.neuropsychoeconomics.org/

The Many Faces of Fear: Attachment, Trauma \& Neuroscience Perspectives October 17-20, 2007; San Diego, CA (USA)

Info: http://www.aapweb.com

Society for Physiology Research, 47th Annual Meeting

October 17-21, 2007; Savannah, GA (USA)

Info: http://www.sprweb.org

ESM'2007 European Simulation and Modelling Conference

October 22-24, 2007; St. Julians (Malta)

Info: http://www.eurosis.org/cms/?q=node/208

Emotions 2007

October 22-24, 2007; Tilburg (Netherlands)

Info: http://www.tilburguniversity.nl/faculties/fsw/ emotions2007/programme/

5th Conference Psychological and pedagogical disciplines in the technical university: methodology, experience, future trends October 25-26, 2007; Kyiv (Ukraine)

Info: http://www.pptu2007.org.ua
Cem07-Cognition, Emotion \& Motivation October 26-28, 2007; Hammamet (Tunisia) Info: http://www.isefc.rnu.tn/cem07/

International Conference on Complex Systems 2007 October 28-November 2, 2007; Boston, MA (USA) Info: http://www.necsi.org/events/iccs7/

International Mind, Brain and Education Society Inaugural Conference

November 1-3, 2007; Fort Worth, TX (USA)

Info: http://www.imbes.org/conference/conf07.html

37th Annual Meeting of the Society for Neuroscience November 3-7, 2007; San Diego, CA (USA)

Info: http://www.apu.sfn.org/

Theory in Cognitive Neuroscience-Cognitive Science and Neuroscience from Conceptual and Theoretical Perspectives

November 4-7, 2007; Wildbad Kreuth (Germany)

Info: http://www.upd.unibe.ch/research/symposien/ HA14.html

7th International Conference on Epigenetic Robotics November 5-7, 2007; Piscataway, NJ (USA)

Info: http://www.d.umn.edu/ cprince/Conferences/ EpiRob07/

Evaluation 2007 Annual Conference November 7-10, 2007; Baltimore, Maryland (USA) Info: http://www.eval.org/eval2007/

Society for Phenomenology and the Human Sciences November 8-10, 2007; Chicago, IL (USA)

Info: http://www.pages.slu.edu/faculty/harriss3/SPHS/ conference.html

AAAI Fall Symposium Series November 8-11, 2007; Arlington, VA (USA) Info: http://www.aaai.org/Symposia/Fall/2007/fss-07.php

Hybrid Artificial Intelligence Systems Workshop November 12-13, 2007; Salamanca (Spain) Info: http://www2.ubu.es/hais2007/

48th Psychonomic Society Annual Meeting November 15-18, 2007; Long Beach, CA (USA) Info: http://www.psychonomic.org/meet.htm

1st International Conference on Cognitive Neurodynamics (ICCN'07) 3rd Shanghai International Conference on Physiological Biophysics (SICPB'07) November 17-21, 2007; Shanghai (China) Info: http://www.iccn2007.org 
The Fourth International Conference on Computational Intelligence, Robotics and Autonomous Systems

November 28-30, 2007; Palmerston North, New Zealand

Info: http://www.ciras.massey.ac.nz/

International Conference on Intelligent

\& Advanced Systems

November 28-December 1, 2007; Kuala Lumpur

(Malaysia)

Info: http://www.icias2007@yahoo.com

World Psychiatric Association International

Congress 2007

November 28-December 2, 2007; Melbourne

(Australia)

Info: http://www.wpa2007melbourne.com

International Conference on Ergonomics

2007 (ICE2007)

December 3-5, 2007; Penang (Malaysia)

Info: http://www.ice2007.um.edu.my

International Society for Quality-of-Life Studies (ISQOLS): From QOL Concepts to QOL

Performance Measures

December 6-9, 2007; San Diego, CA (USA)

Info: http://www.isqols.org/

IADIS International Conference on Cognition and Exploratory Learning in Digital Age

(CELDA 2007)

December 7-9, 2007; Algarve (Portugal)

Info: http://www.celda-conf.org/

6th WSEAS International Conference on

Computational Intelligence, Man-Machine Systems and Cybernetics (CIMMACS '07)

December 14-16, 2007; Tenerife, Canary Islands (Spain)

Info: http://www.wseas.org

3rd Indian International Conference on Artificial Intelligence (IICAI-07)

December 17-19, 2007; Pune (India)

Info: http://www.iiconference.org

2008

2nd International Workshop on Bioinformatics (IWOBI '08)

February 5-8, 2008; Santa Clara, Villa Clara (Cuba)

Info: http://www.iwobi.uclv.edu.cu
International Neuropsychological Society (INS)

Annual Conference

February 6-9, 2008; Waikoloa, Hawaii (USA)

Info: http://www.the-ins.org/meetings

Society for Cross-Cultural Research, and Society for Anthropological Sciences Joint Annual Conference February 20-23, 2008; New Orleans, Louisiana (USA)

Info: http://www.meeting.sccr.org

The First Conference on Artificial General Intelligence (AGI-08)

March 1-3, 2008; Memphis, TN (USA)

Info: http://www.agi-08.org/

Philosophy and Science: Contemporary Explorations March 13, 2008; Raleigh, NC (USA)

Info: http://www.david.snu.edu/ brint.fs/wpsjnl/

Integrative Approaches to Affective Disorders March 14-17, 2008; Cape Town (South Africa)

Info: http://www.isad.org.uk

AAAI 2008 Spring Symposia

March 24-26, 2008; Stanford, CA (USA)

Info: http://www.aaai.org/Symposia/Spring/sss08.php

International Conference on Infant Studies

March 26-29, 2008; Vancouver, BC (Canada)

Info: http://www.isisweb.org

16th European Congress of Psychiatry

April 5-9, 2008; Nice (France)

Info: http://www.kenes.com/aep/

Annual Meeting of the American Psychiatric

Association

May 3, 2008; Washington, DC (USA)

Info: http://www.psych.org

3rd Biennial Meeting of the EARLI Special Interest Group 16-Metacognition

May 8-10, 2008; Ioannina (Greece)

Info: http://www.sig16.uoi.gr

20th Annual Convention, Association for

Psychological Science

May 22-25, 2008; Chicago, IL (USA)

Info: http://www.psychologicalscience.org/convention/

Advanced Visual Interfaces (AVI 2008) International Working Conference

May 28-30, 2008; Napoli (Italy)

Info: http://www.hci.uniroma1.it/avi2008/index.html 
Third International Conference on Cognitive Science (Biennale of Cognitive Science 2008)

June 20-25, 2008; Moscow (Russia)

Info: http://www.cogsci2008.ru/eng/

International Association for Research in Economic Psychology (IAREP) Annual Conference

July 5-8, 2008; Paris (France)

Info: http://www.team.univ-paris1.fr/iarep-sabe2006/

Third International Conference on the Teaching of Psychology

July 12-16, 2008; Saint-Petersburg (Russia)

Info: http://www.ictp-2008.spb.ru/

23rd AAAI Conference on Artificial Intelligence (AAAI-08)

July 13-17, 2008; Chicago, IL (USA)

Info: http://www.aaai.org/Conferences/AAAI

6th International Conference, International Test Commission: The Public Face of Testing July 14-16, 2008; Liverpool (UK)

Info: http://www.intestcom.org

14th European Conference on Personality

July 16-20, 2008; Tartu (Estonia)

Info: http://www.ecp14.ee
Sixth International Conference on Emotions and Organizational Life 'EMONET VI'

July 17-19, 2008; Fontainbleau (France)

Info: http://www.uq.edu.au/emonet/

29th International Congress of Psychology

July 20-25, 2008; Berlin (Germany)

Info: http://www.icp2008.de

19th Congress of the International Association for Cross-Cultural Psychology

July 27-31, 2008; Bremen (Germany)

Info: http://www.iu-bremen.de/iaccp2008/

116th Annual Convention of the American Psychological Association

August 14-17, 2008; Boston, MA (USA)

Info: http://www.apa.org/convention

14th World Congress of Psychophysiology (IOP2008) September 8-13, 2008; St. Petersburg (Russia)

Info: http://www.world-psychophysiology.org/iop2008

14th World Congress of Psychiatry

September 20-25, 2008; Prague (Czech Republic)

Info: http://www.wpa-prague2008.cz 